\title{
Effect of Buteyko breathing exercise in newly diagnosed asthmatic patients
}

Prasanna K. B., Sowmiya K. R. ${ }^{1}$, Dhileeban C. M. ${ }^{2}$

Departments of Medicine, ${ }^{1}$ Community Medicine and ${ }^{2}$ Tagore Medical College and Hospital, Rathinamangalam, Chennai, Tamil Nadu, India

Address for the Correspondence: Dr. K. B. Prasanna, Department of Medicine, Tagore Medical College and Hospital, Rathinamangalam, Chennai - 600 127, Tamil Nadu, India. E-mail: krs3012@gmail.com

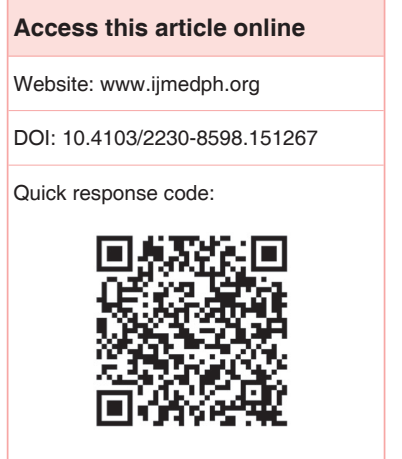

Introduction: Asthma is a chronic inflammatory disease of the airways and is on the increasing trend owing to air pollution and urbanization. The use of alternative ways of treatment that are as efficacious as the standard treatment is the need of the hour. This study was conducted to study the effects of Buteyko breathing exercise on the newly diagnosed asthmatic patients. Materials and Methods: Totally, 100 newly diagnosed asthmatic patients attending Tagore Medical college out-patient department were randomly allocated into the interventional and the control group for a period of 2 months. The former were taught Buteyko breathing exercise while the control group was on standard asthma treatment. The effects were assessed using a pretested close ended Asthma Control Questionnaire and Pulmonary Function Tests. The results were given in mean with a standard deviation. Chi-square test was done to compare the results between the two groups and $P<0.05$ is taken as significant. The analysis was done by SPSS software version 13. Results: Among the 100 participants, majority of the participants were in the age group of 31-40 years. It was observed that there was an overall subjective improvement of asthma symptoms among the interventional group at the end of 2 months (which was statistically significant) when compared to the control group. Also, there was an improvement in the pulmonary function in terms of peak expiratory flow rate in both the groups. Conclusions: The results of this study support the effectiveness of Buteyko breathing exercise over the standard treatment in the newly diagnosed asthmatic patients.

Key words: Alternative treatment, asthma, pulmonary function test

\section{INTRODUCTION}

Asthma is a chronic inflammatory disorder of the airways that causes recurrent attacks of breathlessness wheezing, chest tightness and cough particularly at night or in the early morning. It is mainly due to airway hyper responsiveness to a variety of stimuli resulting in inflammation of the bronchial wall and increased mucus secretion thereby causing broncho constriction and airflow limitation. ${ }^{[1]}$ Different kinds of individuals react differently to various triggering factors. ${ }^{[2]}$ The Main Causes for Asthma are house dust mites in bedding, carpets and stuffed furniture, pollution, pet dander, tobacco smoke, chemical irritants in the workplace and air pollution. ${ }^{[3]}$ Asthma can be differentiated from chronic obstructive pulmonary disease and other respiratory infectious disease with the help of Spirometry and peak expiratory flow meter. ${ }^{[4-6]}$ Severe and uncontrolled asthma can be life threatening and its incidence has increased significantly since late 1970s. Many drugs are used in the management of asthmatic patients. However, they are not found to improve the quality of life adequately. In addition, they also have serious adverse effect which limits their long term use. To combat these problems, we considered using the Buteyko method widely practiced in Russian asthmatic population. ${ }^{[7]}$

Buteyko method was discovered by Russian clinical physician, Mr. Konstantin Pavlovich Buteyko, MD, PhD. Buteyko therapy aimed to reduce chronic hyperventilation. ${ }^{[7}$ Buteyko breathing would bypass the adverse effects of steroids, patient's quality of life could be improved and most importantly this would be cost-effective. Also, the patient compliance could be better than steroids. ${ }^{[8]}$ Hence, this study was done to compare the effects of an alternative way of treatment over the high-cost steroid management which also has many adverse effects. 


\section{Aims}

To assess the effects of Buteyko breathing exercise in the newly diagnosed asthmatic patients.

To note the improvement in their symptoms in patients undergoing Buteyko breathing exercise.

\section{MATERIALS AND METHODS}

The study was conducted in Tagore Medical College and Hospital. Totally, 100 newly diagnosed asthmatic patients attending the medicine out-patient department (OPD) of Tagore Medical College, and Hospital was randomly selected for the study. The study was conducted for 2 months (June 2013 and July 2013). Patients of both sexes in the age group of 25-60 years were selected. Smokers and chronic asthmatic were excluded. Written consent was obtained from all the participants, and the Institutional Ethics Committee approved the study.

This study had a parallel group design with the participants being randomly allocated into the interventional group who were taught Buteyko breathing exercise and the other group was put on inhaled corticosteroids (ICS). The Buteyko breathing exercise was demonstrated to the interventional group. They were instructed to do the breathing exercise at least twice in a day (Morning and Evening). The regular follow-up was done through frequent phone calls. Also the participants were asked to visit the hospital weekly once for assessing their well-being and getting their feedback. The effects of the intervention were assessed by:

1. Their improvement in their pulmonary function-forced expiratory volume in $1 \mathrm{~s}$ (FEV1) and peak expiratory flow rate (PEFR) at the beginning and at the end of $1^{\text {st }}$ and $2^{\text {nd }}$ month of the study using peak expiratory flow meter and Pulmonary Function Test.

2. Subjective improvements of symptoms at the beginning and at the end of $1^{\text {st }}$ and $2^{\text {nd }}$ month of the study using a pretested, structured, close-ended questionnaire in the local language. The questionnaire assessed the severity of symptoms, frequency of exacerbations, changes in the lung capacity, and side effects of medication. The Asthma control scoring was divided on the basis of the total score into Good $(\leq 15)$, Better (16-20) and Poor (21-30).

\section{Buteyko breathing steps}

The first thing to remember when practicing Buteyko style breathing is to breath in a very controlled and shallow manner air should not be sucked like your last breath, it should be a gentle rhythm of breathing in and out. ${ }^{[7,9,10]}$

\section{Step 1}

Keep your mouth closed at all times and only use your nose to breath. It may be hard if you have a blocked nose, but it should eventually clear once you start the breathing technique.

\section{Step 2}

Buteyko breathing requires that you breathe into your diaphragm (stomach) and not your chest. In fact, some may notice Buteyko is similar to some meditation style breathing techniques. When breathing check to make sure your diaphragm inflates and deflates in a controlled manner and your chest remains still.

\section{Step 3}

When breathing ensures you breathe in a very shallow manner so if, you help a blade of grass under your nose, it would barely move. This shallow controlled breathing also applies for the breathing in part.

\section{Step 4}

Sit in an upright position and shallow breath for around 2-3 min, also remember to breathe in fully and do not do partial inhales.

After the 2-3 min period when you get to the exhaling part of your breathing, pinch your nose closed and pause your breathing until you feel the urge to breath.

For beginners, this may be as quick as a few seconds but your aim as your progress is to be able to hold your breath for around a minute.

\section{Step 5}

After holding your breath for a comfortable amount of time, un-pinch your nose and resist the urge to draw in a big breath of air instead continue with the shallow breathing technique. It is hard at first and if you find it difficult you may have held your breath for too long.

\section{Data analysis}

Data were entered in Excel Sheet (Microsoft Corporation). The results were given in mean with a standard deviation. Chi-square test was done to compare the results between the two groups. $P<0.05$ is taken as significant. The analysis was done by SPSS software version 13 (SPSS Inc., Chicago).

\section{RESULTS}

Among the 100 participants, majority of the participants were in the age group of $31-40$ years. The mean age of the subjects in the study group was $40.4 \pm 9.1$ years and in the control group it was $37.36 \pm 7.9$ years [Figure 1].

Females formed the major proportion in both the interventional and control group. In the interventional group, females constituted $64 \%$ and in the control group females constituted $60 \%$ of the study population [Figure 2].

It was seen that asthma control was poor in both interventional and control groups at the beginning of the study [Figure 3].

After starting Buteyko exercise, in both the groups, there was an improvement in the daily asthma symptoms control for some of the patients. Figure 4 illustrates the Asthma control of both interventional and control groups at the end of the study period of 2 months. There was a good improvement in their Asthma control.

The daily asthma control scoring of the subjects in both the groups were as follow, In the interventional group, 29 fell in the Good category, 19 in Better and 2 were in the Poor category. 


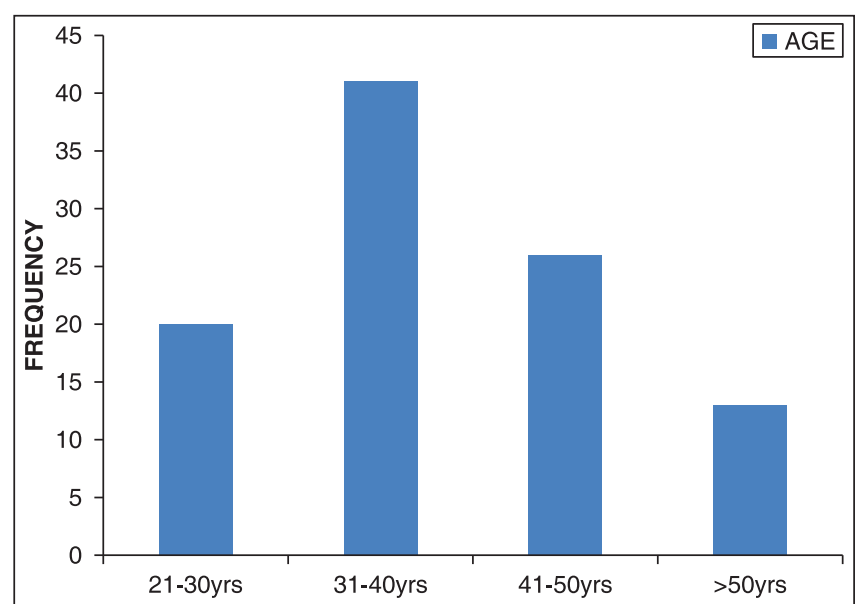

Figure 1: Age distribution

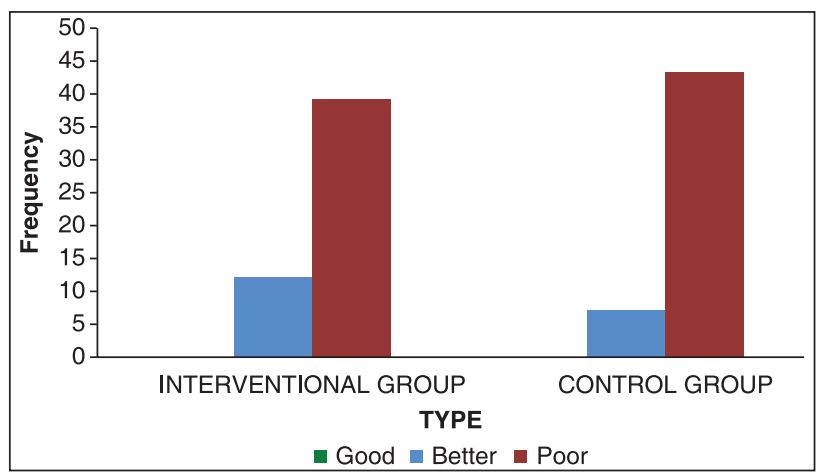

Figure 3: Asthma control at the beginning of the study

And in the control group the number of subjects in each group was: Good-24, better-20, poor-6.

It is evident from the above figures that there was an overall subjective improvement of asthma symptoms. And there was a statistically significant difference in the Asthma Control between the interventional and control group $(P<0.05)$. The month-wise improvement in the Asthma control in the interventional group is shown in Figure 5.

It was seen in our study that the individuals in the age group of 31-40 years had a better improvement over their asthma control at the end of 2 months (which was highly statistically significant) when compared to the other age groups.

There was an improvement in the pulmonary function in terms of PEFR in both the groups as seen in Figure 6.

There was an increase in PEFR values for $56 \%$ of people in the interventional group and $30 \%$ in the control group. Table 1 gives the PEFR of the interventional group before and after the treatment, and this improvement was also statistically significant $(P<0.05)$. The increase in FEV1 was $22 \%$ and $20 \%$ in the study and control group respectively and it was not statistically significant $(P>0.05)$.

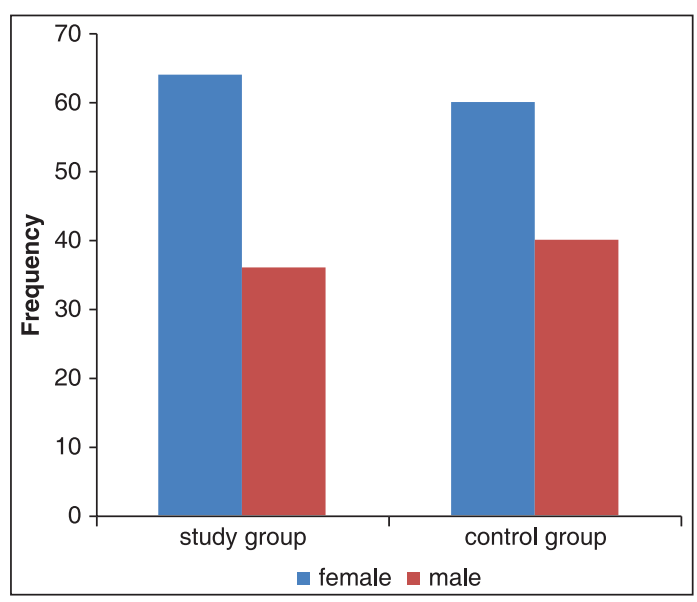

Figure 2: Sex distribution

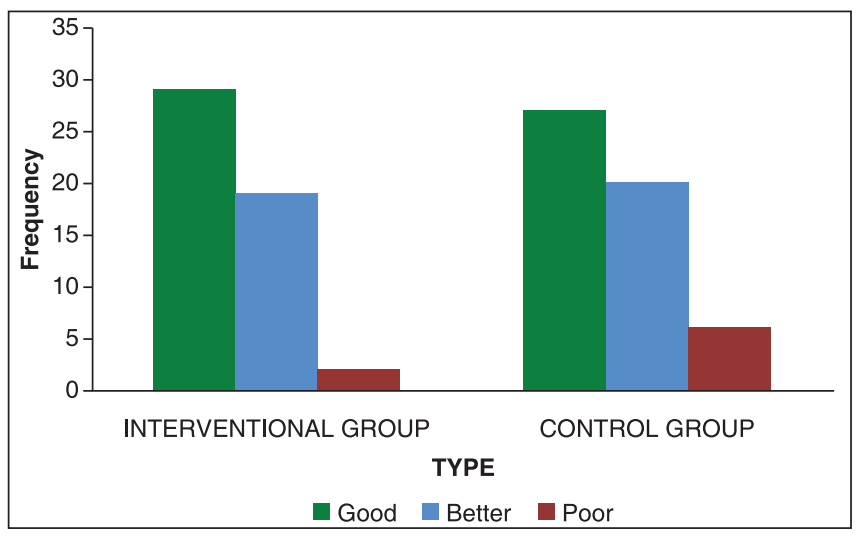

Figure 4: Asthma control at the end of 2 months

\section{DISCUSSION}

This present experimental study was done among 100 newly diagnosed asthmatic patients attending the medicine OPD of Tagore Medical College and hospital in Chennai. The aim of the study is to assess the effects of Buteyko breathing exercise in the newly diagnosed asthmatic patients and note their subjective symptomatic improvement.

In this study, the study population belonged to the age group of 25-60 years similar to the study conducted in South Brisbane in which the subjects were aged between 25 and 70 years. ${ }^{[9]}$ In a study conducted by Hassan et al. in Egypt, the study subjects were 18 females and 22 males in the age group of 30-50 years with a mean age of $42.2 \pm 7.12$ years. ${ }^{[1]]}$ But in the present study, there was an overrepresentation of females. They constituted around 64\% of the study group and $60 \%$ of the control group. Also, the mean age of the study population in the present study was $40.4 \pm 9.1$ years.

In a study done by Cooper et al., the study subjects were all nonsmokers similar to the present study in which the subjects were also nonsmokers. ${ }^{[12]}$

The study subjects were enrolled in the study for a period of 2 months during which they were followed-up thrice a week. 


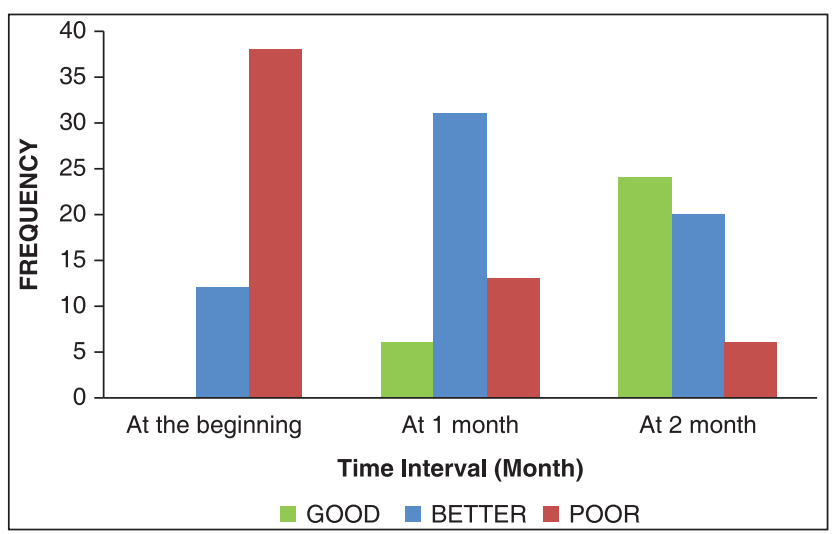

Figure 5: Asthma control among the interventional group during the study period

\begin{tabular}{|c|c|c|}
\hline & Pretreatment & Post treatment \\
\hline Mean $\pm S D$ & $209.4 \pm 66.59$ & $291.8 \pm 73.309$ \\
\hline Mean difference & 82.4 & \\
\hline$P$ & 0.000 & \\
\hline
\end{tabular}

McHugh et al., in his study that was conducted for 6 months, the patients were followed-up by phone every 3 weeks. ${ }^{[8]}$

In an old study done by Buteyko and Genina which included 52 children with regular asthma attacks, he observed over a period of 1-3 months that the people who did Buteyko breathing exercise had 83\% improvement in their daily asthma symptoms. ${ }^{[13]}$ Bowler et al. found out Buteyko breathing technique reduced the hyperventilation and their use of beta 2 -agonists. It causes reduction in the use of inhaled steroids and better quality of life. ${ }^{[9]}$ This was similar to the results obtained in our study where, there was a significant improvement in their daily asthma symptoms among the interventional group thus a better quality of life. Cooper et al. proved that the Buteyko breathing technique can improve symptoms and reduce bronchodilator use. ${ }^{[12]}$ McHugh et al. assessed the improvement by the help of symptoms score, FEV1, medication use and revealed that the Buteyko breathing technique is a safe and efficacious asthma management technique. ${ }^{[8]}$

Over $90 \%$ of 1,00,000 patients in Russia have taken the Buteyko exercise which completely provided relief without further Asthma Medication. Likewise in Australia, 8000 patients have undergone the Buteyko therapy in a successful manner. ${ }^{[7]}$ Cowie applied Buteyko techniques for 6 months and he found that there was improvement in Asthma control from $41 \%$ to $75 \%$, Decrease of ICS by $39 \%$ and Elimination of ICS was $21 \%$. Though the duration of the study was a period of 2 months, it was observed that the improvement in daily asthma symptoms was found to be statistically significant in the interventional when compared to the control groups. If the exercise is continued for a period of 6 months, the effects will have been commendable as with Cowie. ${ }^{[14]}$

Bowler et al. observed an improvement in the PEFR among the participants who were taught Buteyko breathing exercise similar to the present study. ${ }^{[9]}$ In the present study, there was an improvement

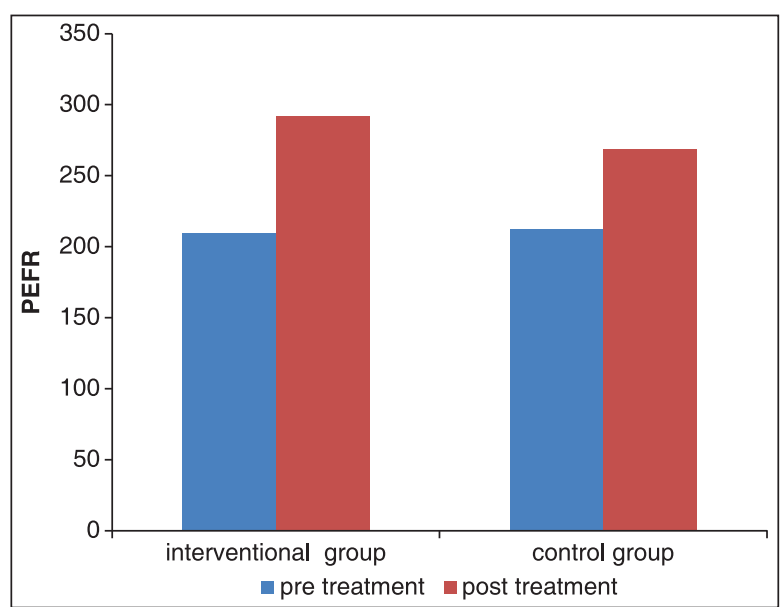

Figure 6: Mean peak expiratory flow rate in both the groups

in PEFR for $56 \%$ of the study subjects. But McHugh et al. recorded no change in PEFR. ${ }^{[9]}$

As per the results, it can be said that:

- Buteyko breathing has a beneficial effect on people living with asthma.

- There is a significant difference in the daily asthma symptoms control between people who take conventional treatment and those who perform Buteyko breathing

- With its effectiveness equivalent to steroids, this Buteyko breathing exercise has the added advantage as it has no side effects, unlike steroids.

The other advantages are as follows:

- Once the exercise is taught to the patient, it can be performed by themselves easily at their homes.

- It can be considered as one of the primary prevention methods for asthma.

- It is a cost effective method compared to the conventional treatment.

- This cost effective method can be practiced even at the primary health care level.

- The Buteyko breathing exercise can be recommended as a primary intervention for the treatment of asthma.

- The judicial use of Buteyko breathing by the medical practitioner to treat asthmatic patients can hence avoid the side effects of steroids.

\section{CONCLUSION}

The results of this study support the effectiveness of Buteyko breathing exercise over the standard treatment in the newly diagnosed asthmatic patients. There was a statistically significant improvement of daily Asthma Control and PEFR in the group who did Buteyko breathing exercise for 2 months over the control group. With an intervention that is safe, equally efficacious and cheap, it is possible to use this Buteyko breathing technique as a primary treatment method in the management of asthmatic patients at the primary health care level itself. 


\section{ACKNOWLEDGMENT}

We are very grateful to ICMR who supported and funded this project under Short Term Studentship (STS 2013) program.

\section{REFERENCES}

1. Kobzik L. The lung — bronchial asthma. In: Robbins Pathologic Basis of Disease, ed. Cotran RS, Kumar V, Collins T, $6^{\text {th }}$ ed., p. 712-6.

2. Baxi SN, Phipatanakul W. The role of allergen exposure and avoidance in asthma. Adolesc Med State Art Rev 2010;21:57-71.

3. Fact sheet: Chronic obstructive pulmonary disease (COPD). World health organisation. Reviewed October 2013; Available from: http://www.who.int/ mediacentre/factsheets/fs315/en/. [Last accessed on 2013 Jan 11].

4. Pierce RJ, Allen CJ, Campbell AH. A comparative study of atropine methonitrate, salbutamol, and their combination in airways obstruction. Thorax 1979;34:45-50.

5. Smith JA, Weber RW, Nelson HS. Theophylline and aerosolized terbutaline in the treatment of bronchial asthma. Double-blind comparison of optimal doses. Chest 1980;78:816-8.

6. Al-Ashkar F, Mehra R, Mazzone PJ. Interpreting pulmonary function tests: Recognize the pattern, and the diagnosis will follow. Cleve Clin J Med 2003;70:866, 868, 871-3.
7. Buteyko Breathing Centre. Available from: http://www.buteyko.co.uk/ trials.html. [Last accessed on 2013 Jan 23].

8. McHugh P, Aitcheson F, Duncan B, Houghton F. Buteyko Breathing Technique for asthma: An effective intervention. N Z Med J 2003;116:U710.

9. Bowler SD, Green A, Mitchell CA. Buteyko breathing techniques in asthma: A blinded randomised controlled trial. Med J Aust 1998;169:575-8.

10. Stalmatski A. Freedom from asthma: Buteyko's revolutionary treatment. Hale Clinic Health Library. London: Kyle Cathie Ltd.; 1997. p. 175.

11. Hassan ZM, Riad NM, Ahmed FH. Effect of Buteyko breathing technique on patients with bronchial asthma. Egypt J Chest Dis Tuberc 2012;6: 235-41.

12. Cooper S, Oborne J, Newton S, Harrison V, Thompson Coon J, Lewis S et al. Effect of two breathing exercises (Buteyko and pranayama) in asthma: A randomised controlled trial. Thorax 2003;58:674-9.

13. Buteyko V KP, Genina A. The results of the BVB method trial. E.M. Sechenov's medical institute, Moscow. Available from: http//www.buteyko. com. [Last accessed on 2013 Jan 10].

14. Cowie R. Buteyko breathing technique, medical trial foothills hospital medical trial. Vol. 3. Calgary, Alberta: The American Thoracic Society; 2006. p. A530.

How to cite this article: Prasanna KB, Sowmiya KR, Dhileeban CM. Effect of Buteyko breathing exercise in newly diagnosed asthmatic patients. Int J Med Public Health 2015;5:77-81.

Source of Support: Nil, Conflict of Interest: None declared.

Announcement

\section{iPhone App}

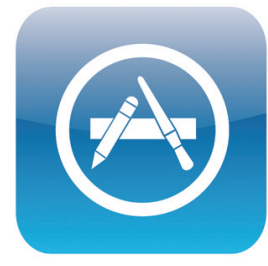

A free application to browse and search the journal's content is now available for iPhone/iPad. The application provides "Table of Contents" of the latest issues, which are stored on the device for future offline browsing. Internet connection is required to access the back issues and search facility. The application is Compatible with iPhone, iPod touch, and iPad and Requires iOS 3.1 or later. The application can be downloaded from http:// itunes.apple.com/us/app/medknow-journals/id458064375?ls $=18 \mathrm{mt}=8$. For suggestions and comments do write back to us. 